medicina novoantiqua ${ }^{93}$, où le neuf est si sûrement bâti sur l'ancien qu'il paraît en émaner.

Ainsi, la Renaissance médicale n'a vu ni un simple retour aux anciens, ni une révolution. Les dates de 1543 et de 1816 circonscrivent à la fois la dernière période de la tradition hippocratique et galénique et la dernière période productive de cette tradition. Cessons donc de ne considérer les découvertes des grands Renaissants qu'au point de vue de la médecine moderne et expérimentale, qui les a incorporées de gré ou de force; apprenons à les envisager aussi en fonction de cette doctrine hippocratique et galénique qui a été leur premier lit.

\title{
Célébrités médicales
}

\section{Par Edgar Goldschmid, Lausanne}

Il n'est pas facile de comprendre pour quelles raisons une renommée pâlit ou s'efface, ni de prévoir les circonstances dont dépendra sa durée. Nous ne pensons pas ici à la vogue soudaine d'une personne ou d'une chose; mais nous voudrions chercher pourquoi, dans l'histoire de la médecine notamment, certaines gloires qui semblaient destinées à vivre toujours, ont disparu.

Prenons par exemple le nom de Sir Charles Bell (1774 - 1842), le grand physiologiste qui brillait aussi dans l'anatomie et la chirurgie: il est presque complètement oublié, et pourtant c'est lui qui découvrit la différence des racines nerveuses, et son nom fut donné à des nerfs et des maladies nerveuses. Sir Charles Bell, qui était conscient de sa valeur et de son importance, se rangeait lui-même aux côtés de William Harvey (1578-1657). Il est mort trop tôt pour avoir pu faire école, étant rentré en Ecosse à un âge relativement avancé. Nous nous demandons également comment il se fait qu'un grand chirurgien tel que Sir Benjamin Brodie (1783-1862), le prétendu successeur de Sir Astley Cooper, connu surtout par ses recherches sur la chirurgie des articulations, soit tombé dans l'oubli après une longue vie qui l'a mené de succès en succès en chirurgie, après des débuts en anatomie. On voudrait savoir aussi pourquoi dans sa biographie il est toujours parlé de ses intentions de s'occuper enfin de physiologie.

${ }^{93}$ D'après le titre d'un ouvrage du médecin M.B. VALENTINI, paru à Francfort en 1713. 
Un de nos contemporains, Heinrich Quincke (1842-1922), le dernier classique de la médecine, auquel nous devons entre autres la ponction lombaire, était déja complètement oublié lors de son centenaire, bien qu'il soit mort à l'âge avancé de quatre-vingts ans - aussi âgé que Sir BENJAMIN Brodie. Et pourtant, Heinrich Quincke avait fait école, mais presque tous ses disciples directs étaient morts avant lui. On doit constater une fois de plus que, dans les sciences, les "petits-fils» déjà ne se réclament plus de leur «grand-père», à moins qu'il ne soit encore en vie.

Un autre savant a complètement disparu de la mémoire de nos contemporains, bien qu'il fût de formation scientifique internationale et bilingue dans ses publications dont le grand atlas du «Traité d'Anatomie Pathologique» est encore d'usage courant, dans les grands instituts au moins, HermanN LEBert, Berlinois né à Breslau en 1813 et mort à Bex, en Suisse, en 1878. LEBERT n'est pas anglo-saxon comme BELL, il n'a pas toujours vécu au même endroit comme Brodie et n'a pas été aussi exclusivement clinicienphysiologiste que Heinrich Quincke; mais il a pratiqué la chirurgie, la botanique, la zoologie, l'anatomie pathologique, l'histologie pathologique et la médecine générale en Suisse et à Paris, et il a professé la clinique médicale aux universités de Zurich et de Breslau, y prenant la succession de K. Ewald Hasse (1810-1903), et de Јон. LuKas Schönleiv (1793-1884), puis à Breslau celle de ThÉODor von Frerichs (1819-1885).

Le fait d'avoir écrit son autobiographie ne sauvera pas une personnalité médicale de l'oubli. Tous ceux que nous venons de citer en ont rédigé, exception faite pour HeINRICH Quincke qui, apparemment, n'en a pas écrit. Sur Sir Charles Bell, nous sommes amplement renseignés par sa correspondance régulière avec son frère, qui a duré jusqu'a sa mort; Sir BENJAmin Brodie nous a laissé une autobiographie, et de Hermann Lebert, nous possédons au moins des Notices biographiques de sa propre main (1869), une plaquette devenue très rare. Habent sua fata libelli: nous savons tous qu'il y a dans de nombreuses bibliothèques des autobiographies qui n'ont jamais été lues que par leurs auteurs.

On pourrait croire qu'une bibliothèque particulière ou une collection portant le nom de son fondateur-propriétaire devrait assurer sa renommée, mais il n'en est rien! D'habitude, les bibliothèques sont vendues par les héritiers, les collections placées dans des musées; et là, après un temps plus ou moins long, elles perdent jusqu'à leur nom. Il en est ainsi de la collection de Jean-Baptiste Wicar (1762) à Anvers, ou de celle de Al. Charles SauVAGEOT (1779-1860) au Louvre, pour ne citer que des collections connues 
d'un public étendu. Citons encore les collections de John Hunter (1728-1793) à Londres, de son frère William Hunter (1718-1783) à Glasgow, de Sir Charles Bell (1774-1842) à Edimbourg. Les musées portant le nom d'un grand médecin tel que Dupurtren (1777-1835) ou Orfila (1787-1853) à Paris, n'évoquent pas mieux ces grands noms que ne le feraient des monuments funéraires. D'autre part, les noms de deux musées médicaux tels que le «Josephinum» et la «Specola», célèbres entre tous, n'ont pas de rapport avec des médecins; l'un porte le nom de son fondateur, l'Empereur JoSEF II (1741-1790), et l'autre ne fait qu'indiquer l'endroit où se trouve l'Observatoire.

Reste l'œuvre littéraire dont un ouvrage ou l'autre pourra survivre et perpétuer le nom de l'auteur, mais ce n'est là qu'une exception. Nous ne citerons que l'Auscultation médiate de LAËNNEG (1819) pour les Français, la Cellularpathologie, de Virchow (1858) pour les Allemands et, pour les Anglais, les ouvrages de Charles Darwin, The Descent of Man et The Origin of Species (1859). Cette énumération est mince, et d'ailleurs ce serait une erreur de croire que toutes les personnes qui connaissent le titre d'un livre l'ont lu. Pour les ouvrages en latin, il ne nous souvient que du livre de Morgagni, De Causis Morborum per Anatomen Indagatis (1761).

Hippocrate, cité par tous les auteurs, est représenté surtout par les jolis petits recueils des Aphorismes en grec ou en latin en-16 , du $18^{\mathrm{e}}$ siècle, rarement lus parce que nous savons que les caractères en sont trop petits pour nos yeux.

Si le souvenir des distinctions conférées à plusieurs grands médecins est parvenu jusqu'à nous, bien souvent nous ignorons pour quels motifs les gouvernements les ont décernées.

Deux médecins français, le baron Guillaume Dupuytren (1777-1835) que Lisfranc (1790-1846) appelait «le brigand de l'Hôtel-Dieu», et le Dr Louis DÉSIRÉ VÉRon (1798-1867), interne des hôpitaux devenu propriétaire-vendeur de la «Pâte Regnault», directeur de l'Opéra de Paris et propriétaire du «Constitutionnel», sont trop connus et ont été trop discutés pour que nous parlions d'eux dans ces pages. Il en est autrement de LEBERT.

Pour connaître les raisons pour lesquelles Hermann Lebert, jeune savant et praticien d'il y a cent ans, a été distingué, le plus simple était de nous adresser au gouvernement qui l'a décoré et à l'Académie qui lui a accordé des prix. La chose était plus compliquée que nous n'aurions pensé. La chancellerie de la Légion d'Honneur - LEBERT avait reçu le grade de chevalier et d'officier - nous a répondu que seuls les gouvernements faibles 
donnent des explications de leurs faits et gestes et que, pour cette raison, il n'existait pas d'«éloge» de LeBERT! Du reste, toutes les recherches auraient été vaines, les Archives de l'Hôtel de la Légion d'Honneur ayant été ravagées par un incendie. Rentrés bredouille, nous en sommes réduits à des hypothèses quant aux véritables motifs qui avaient pu amener le gouvernement français à décorer un jeune praticien et un savant étranger.

Le jeune LeBert avait aidé à soigner les blessés à Paris lors des combats de rues de 1848 et il avait exprimé en public ses sentiments de sympathie pour la France: peut-être est-ce pour cela qu'il reçut sa première décoration. Quant à la seconde, on peut l'expliquer parce qu'il était demeuré à Paris et avait soigné les malades atteints de choléra-morbus pendant l'épidémie de 1852. Ce qu'il a écrit sur cette épidémie ne suffit pas pour nous renseigner.

Dix ans plus tard, LEBERT a publié des rapports sur le choléra à Zurich, signés de lui-même ou écrits en collaboration avec un assistant. Mais on connaît des lettres qu'un autre de ses assistants (WEGELIN) écrivait à sa famille et d'après lesquelles LEBERT, son chef, alors directeur de la clinique, n'aurait pas séjourné à Zurich pendant cette petite épidémie et serait resté introuvable; par conséquent, tout le travail serait incombé à son seul assistant! D'après tout ce qui nous est connu de la vie de Lebert en Suisse, sachant en outre qu'il avait déjà pris part, comme volontaire, aux échauffourées en Valais, en 1844, nous ne saurions l'accuser de lâcheté. Quant à son assistant, n'a-t-il pas, comme tant de jeunes gens manquant encore d'expérience et de psychologie, jugé trop sévèrement son maître parce qu'il le voyait sous un angle déformé, celui qu'en langue allemande on appelle «Froschperspektive»?

Privé d'informations sur sa croix de la Légion d'Honneur, il nous fallait découvrir pour quelles raisons des Prix Monthyon lui ont été décernés le fondateur de ces prix était Ant. Auguste, baron de Monthyon (1733 à 1820).

L'Institut de France, heureusement, n'a pas été incendié, et les rapports imprimés à l'époque sont tous conservés. Nous avons pu les consulter à l'Académie des Sciences, avec l'aide aimable des secrétaires. Les rapports sur les écrits soumis à l'Académie sont signés des commissions et du rapporteur lui-même. Le seul fait qu'un tel rapport a été présenté suffit à prouver l'excellence du travail.

Nous avons lu les rapports sur les travaux présentés à l'Académie en 1846, 1852, 1862 (dates auxquelles LEBERT sollicita et obtint le prix Monthyon); ils sont approfondis et détaillés. Claude-Françors Lalle- 
mand (1790-1853), Etienne-René-Auguste Serres (1787-1863) et GaBRIEl ANDral (1797-1876) les ont signés pour la commission. Les sommes adjugées sont de fr.fr. 1800, 2000 et 2000 . Il est intéressant de constater qu'en même temps que les travaux de Lebert, ceux du pathologiste J.-B. CRUveilhier (1791-1873) et du clinicien Th. v. Frerichs (1819-1885) ont été à l'examen - Cruveilhier est l'auteur du grand recueil et atlas d'anatomie pathologique qui a précédé LeBERT de quelques années, et Frerichs, à qui il devait succéder à la chaire de clinique médicale, à Breslau.

On pourrait se demander si une célébrité sera plus solidement établie si elle a été acquise de bonne heure ou à l'âge mûr - mais ici encore, nous ne trouvons pas de réponse. Cependant, on peut noter que, lors des échauffourées valaisannes (1840 et 1844), Hermann LeBERT était âgé d'une trentaine d'années; lorsque la croix de chevalier lui a été conférée, il avait trente-six ans, et il reçut la rosette à quarante-six ans. Il avait quarante ans environ quand il reçut le prix Monthyon et quarante-quatre ans lors de la parution de son grand traité d'anatomie pathologique.

Comme on vient de le voir, le prix Monthyon, en plus de la gloire, accordait aux bénéficiaires une certaine somme d'argent qui pouvait leur être fort utile pour la poursuite de leurs recherches scientifiques; ce fut le cas pour LEBERT.

Ceci nous amène à envisager un aspect de la question qui, lui aussi, relèverait plutôt de la sociologie que de la médecine. En étudiant les diverses biographies et autobiographies de médecins, nous remarquons entre elles une différence fortement marquée, principalement entre les écrivains allemands et les anglo-saxons.

Dans les biographies allemandes, il est exceptionnel de trouver des renseignements d'ordre financier; on y mentionne tout au plus quelques indications ou quelque revendication de traitement à accorder par le gouvernement, ou bien encore une tentative (Dieffenbach) de récupérer des honoraires dûs par une cour impériale et détournés par un intermédiaire.

En effet, en Allemagne, la plupart de ces médecins étaient des fonctionnaires d'Etat, même s'ils pratiquaient leur art en dehors du professorat. Il faut toujours se rappeler que ce ne sont pas là des «hommes d'affaires» mais des «professionnels» qui n'attachent pas une importance excessive aux honoraires.

Dans les biographies de langue anglaise, nous nous trouvons en face de médecins qui font partie d'un hôpital privé, qui possèdent une école médicale privée ou y collaborent. Pour la plupart d'entre eux, la clientèle privée 
est l'essentiel, et ceci non pas seulement chez les Américains, comme on pourrait être tenté de le croire, en pensant à la retentissante discussion sur le «full time».

En Angleterre, les revenus d'un médecin jettent une certaine lumière sur sa célébrité. Ce n'est pas seulement dans leur journal ou leur calepin que ces médecins inscrivaient les sommes qu'ils recevaient, mais leurs autobiographies et biographies mentionnent régulièrement la hausse ou la baisse de leur revenu. Ils parlent ouvertement de leur «business » même si, comme Sir Charles Bell, ils préfèrent de beaucoup leur activité scientifique tout-à-fait conforme au dicton anglais "politics is business ». Une tabatière royale, c'est très bien, de la vaisselle plate, c'est très utile. "I don't know who you are", dira Sir CHARLEs, "but I want the rich to pay for the poor." Il fut d'ailleurs réduit à vendre sa collection parce que son revenu était insuffisant.

A la même époque, nous trouvons dans la correspondance des frères Hunter la charmante lettre de John (1728-1793) à William (1718-1783) pour introduire auprès de lui un malade qui avait la bourse plate: «Il n'a pas d'argent, et toi, il ne t'en faut pas - vous voilà donc bien assortis.»

Nous savons que Sir Astley Cooper (1768-1841), après avoir joui d'un revenu fabuleux ( $£ 20000$ par an), se retira à la campagne. Il racontera comment, rongé par l'ennui, il contemplait les arbres de son parc pour choisir celui qui lui servirait à se pendre. Bientôt, il n'y.tint plus et s'en retourna à la ville retrouver ses malades. Lui-même nous dira qu'il préférait le paiement comptant aux billets de banque envoyés par lettre, parce que ce dernier mode de paiement lui faisait perdre le shilling qui distingue la guinée de la livre.

Sir Benjamin Brodie, l'auteur célèbre des Maladies des articulations, qu'on a l'habitude de désigner comme le successeur de Sir Astrey Cooper, avait un revenu de $£ 10000$ au moins, accumulé guinée après guinée, comme le constate GARRISON, qui n'oublie jamais de nous informer de pareils détails. Mais son biographe, Tim. Holmes, fera de son mieux pour affirmer à toute occasion que Sir BENJAMIN ne s'intéressait pas à l'argent, sans pourtant oublier de rappeler combien il était renseigné sur les affaires financières! Nous connaissons ce fait charmant que Sir BEnJAmin a invité et soigné à ses frais un étudiant dans sa propre maison de campagne. Mais le biographe dira encore que Sir BENJAMIN préférait les placements en propriétés foncières; celle qu'il a acquise en dernier lieu avait une superficie de quelque 450 acres - Regent's Park à Londres n'en a que 472! Comme il possédait 
déjà plusieurs fermes dans le Suffolk, Sir Benjamin savait au moins où trouver le repos le jour où il se retirerait de la vie active et quitterait la ville.

Le récit d'une discussion à l'époque napoléonienne pourra nous renseigner sur les appointements touchés par les médecins qui venaient pratiquer aux cours de France ou de Russie. Jean-Nicolas Corvisart (1755-1821), peu enchanté de voir surgir un nouveau concurrent éventuel, demandait insolemment à Johann Peter Frank (1745-1821) s'il voulait vraiment donner suite à la demande de NAPOLÉon et entrer à son service. Frank répondit que Corvisart, apparemment, se méprenait sur la personne du «Vieux Frank »: «Bien entendu, vous êtes le Premier médecin de l'Empereur avec un traitement de 30000 francs; mais moi, comme Conseiller d'Etat à Saint Pétersbourg, j'y touchais déjà mes 42000 roubles » (ce qui représente à peu près le triple de la somme en francs français à fr.fr. or $=2.80$ ).» «Eh bien, j'en parlerai à l'Empereur.»

Quant aux médecins Hollandais du début du XVIII' siècle, rappelons que le plus célèbre d'entre eux, le grand BoErhaAve - le "précepteur du monde» (1668-1738) - fils d'un pasteur de Leyde, laissa une fortune princière, évaluée à deux millions de florins. C'est là une des rares indications de cet ordre que nous puissions trouver sur les Hollandais. Elle se lit dans les Anecdotes de l'Histoire Médicale du monde par Eugéne Hollaender, qui nous dit encore de cette fortune, «ein nicht zu unterschätzender Gradmesser seiner ärztlichen Bedeutung» ... une formule qui nous permet de mesurer le degré de son importance médicale.

Mais toute cette célébrité de leur vivant n'a pas sauvé de l'oubli ces grands médecins et savants après leur mort, en dépit de l'aphorisme célèbre de BALZAC (1799-1850) : «La gloire est le soleil des morts.»

A la mort de RoDolphe Virchow (1821-1902), décédé à un âge avancé, son nom a disparu tout d'un coup des travaux publiés et des discussions des sociétés médicales bien que, pendant près d'un demi-siècle, il y eut tenu la première place et que son apport aux sciences médicales ait été incomparable ${ }^{1}$. C'est à peu près le même phénomène qui s'était produit à la mort de GoEthe (1749-1832) : les uns considéraient la mort de «l'Olympien» comme une catastrophe nationale, tandis que les autres se sentaient soulagés d'un poids insupportable à la disparition de «Son Excellence».

Ce titre d'«Excellence», comparable au titre français de «Baron», con-

${ }^{1}$ Pour rafraîchir sa mémoire, la génération actuelle n'aura qu'à jeter un coup d'œil sur l'article de Scheuthauer dans le Lexique biographique des Médecins, paru en 1884 ss. 
féré, dans la hiérarchie allemande, aux sommités scientifiques, n'est pas dépourvu d'un certain comique, au moins aux yeux des jeunes. Pour s'adresser au vieux chirurgien ERnst von BergmanN, un médecin berlinois dira "von unseres Herrn Vorsitzenden Excellenz», langage qui peut paraître servile, mais qui est simplement celui de l'époque. Ouvrez donc le fameux Wunderhorn publié par L. Achim von Arnim (1781-1831) et Clemens Brentano (1778-1842), deux représentants de l'époque dite romantique, en 1806, et vous trouverez la dédicace «Seiner Excellenz des Herrn Geheimerath von GoETHE » - offerte au poète qui n'avait alors que cinquantesept ans.

Un autre genre de célébrité est celle du Dr Ernst Ludwig Heim (1747 à 1834), Thuringien, médecin à Spandau et à Berlin. Si nous ne faisons erreur, il y a trois médecins dont le nom est resté vraiment populaire en Allemagne: Paracelse, Eisenbart (1671-1761) et «der alte Heim». Heim n'était pas un «scientifique», il a très peu écrit, mais il était protagoniste de la vaccination et le praticien le plus recherché de la région de Berlin. Adoré de ses malades, il jouissait d'un caractère doux et charitable et nombreux sont les bons mots et les boutades qu'on lui prête. Sa clientèle ne lui rapportait, à partir de 1800, que 36000 marks environ. Bien que ce fût une grosse somme dans les circonstances où se trouvait l'Allemagne à cette époque, elle paraît modeste en comparaison de ce que gagnaient les Anglais et les Français et surtout les médecins appelés à la cour de Cathérine II (1729-1796) par exemple. Pour donner une idée du personnage, disons que Heim faisait sa ronde de visites médicales à cheval, suivait ses malades en les regardant par la fenêtre, et qu'il élevait une famille. Au besoin, il faisait lui-même l'autopsie de ses enfants, aidé par le valet de l'équarrisseur, parce qu'il n'y avait pas, à cette époque, d'instituts de pathologie.

Comme celui de Heim, le nom d'un autre chirurgien célèbre, JoH. FriedRigh Dieffenbach (1792-1847), Professeur à Kœnigsberg et à Berlin, est resté gravé dans la mémoire de la population berlinoise, autant pour ses grands succès en chirurgie plastique et générale et sa personnalité captivante, que pour certains détails de son train de vie. Ses contemporains vantaient la beauté de ses «carrossiers» qu'il conduisait lui-même, quand il allait visiter ses malades en ville. Quelle différence avec le mode de locomotion de Sam. Dav. Gross de Philadelphie (1805-1884), qui préférait pour vaquer à sa pratique médicale, "a buggy and a respectable horse". Bien longtemps encore après la mort de ce chirurgien si populaire, les enfants dans les rues de Berlin chantaient: 


\section{«Das ist der Doktor Dieffenbach, Professor wohlgeboren, Er schneidet Arm' und Beine ab, Macht neue Nas' und Ohren.»}

La teneur de ces vers étant quelque peu sommaire, il vaut la peine de rappeler les paroles de Dieffensach pour saluer l'invention de l'anesthésie chirurgicale: «Der schöne Traum, daß der Schmerz von uns genommen, ist zur Wahrheit geworden» (Le beau rêve d'être délivrés de la douleur s'est réalisé).

Par comparaison, il ne sera pas déplacé de citer ici la boutade de MagenDIE (1783-1855) à la même occasion, telle que Monsieur Mondor nous la transmet: "Qu'un malade souffre plus ou moins, est-ce là une chose qui offre de l'intérêt pour l'Académie des Sciences ?»

C'est à cette époque que JACoB BurGKhardt faisait ses études à Berlin, où l'on discutait les médecins de la Cour Royale, entre autres LuGAs SchöNLEIN, le réformateur de la clinique allemande. Lorsque SchöNLEIN (1793 à 1864) fut appelé comme médecin ordinaire du prince héritier de Prusse (plus tard Frédéric Guillaume IV), Jacob Burckhardt écrivit à Bâle²: «... Même ces «grands Seigneurs» sont loin d'avoir des revenus aussi considérables que nos messieurs de Bâle, et Monsieur Christoph Merian (1800-1858) jouit d'un revenu bien plus important que le Prince Royal de Prusse, car celui-ci ne touche qu'une rente annuelle de Fr. 270000, avec laquelle il doit entretenir une foule de gens, tandis que Monsieur MÉrian peut probablement mettre de côté la même somme.»

Pour terminer notre tour d'horizon, il nous paraît intéressant, instructif et même amusant de jeter encore un coup d'œil sur les médecins de la Société des Amis, les Quakers, médecins scientifiques et praticiens très en vogue. Quiconque désire les connaître d'une façon plus approfondie, aura recours à l'excellent livre de Hingston Fox sur John Fothergill et ses Amis (1919).

A Londres, John Fothergill (1712-1780) était un médecin Quaker jouissant d'une très grande renommée dans divers domaines. Son jardin botanique fut l'objet de l'admiration de plusieurs générations. Ses splendides collections de coquilles, d'insectes et de dessins ont maintenant été jointes à celles de William Hunter et léguées à l'Université de Glasgow.

${ }^{2}$ JacoB Burckhardt nach W. Loeffler, Med. Klin. Zürich, p. 40. 
Fothergill n'est pas oublié, car son nom, avec celui de LetTsom, est resté vivant grâce à la Société médicale de Londres, où existent encore à l'heure actuelle les fondations pour les Conférences Fothergill et Lettsom. La participation de Fothergill, un homme connu pour sa philanthropie, à la fondation du Pennsylvania Hospital, est présente à toutes les mémoires aux Etats-Unis, tout comme on se souvient encore des efforts de cet ami de Benjamin Franklin pour réconcilier l'Angleterre et les Etats-Unis.

Les Fothergill, du reste, ne sont pas éteints. John Fothergill, notre contemporain, descend de l'illustre médecin et naturaliste et mesure parfaitement la valeur de ses ancêtres. Etant archéologue, il a dû changer de métier après la première guerre mondiale; il s'est fait «aubergiste» et nous a gratifié d'un charmant petit livre, An Innkeeper's Diary.

Un des Quakers les plus renommés vers la fin du XVIII ${ }^{\mathrm{e}}$ siècle, était JoHn Coakley Lettsom (1744-1815), né aux Virgin Islands, co-fondateur de la Société médicale de Londres (1773), auteur d'une captivante Histoire des Origines de la Médecine (1778). Comme «Tout finit par des chansons», ${ }^{3}$ ce sont surtout des vers railleurs qui nous rappellent son existence, et encore des vers d'expression quelque peu vulgaire - provoqués peut-être par l'importance de ses revenus qui atteignaient en moyenne $£ 12000$ par an. Il en disposait, tout comme le faisait Fothergill, "prodigue en dépense, généreux en philanthropie» - contentons-nous de ne citer qu'un seul quatrain parmi tant d'autres qu'on trouve par exemple dans la publication de F. Parkes Weber:

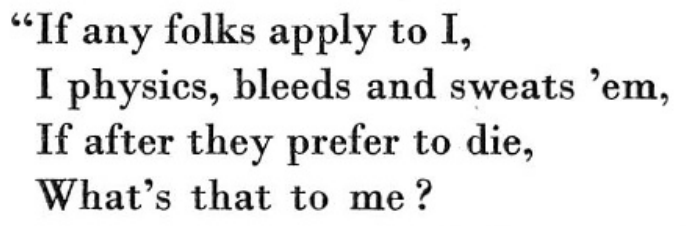

\section{LetTsom."}

Pour terminer, nous rappellerons trois gloires médicales de l'antiquité, dont le souvenir a été conservé vivant par la littérature comme par la sculpture, depuis bien des siècles. Les plus éloignés de nous, engloutis dans les ténèbres de la préhistoire, sont le centaure Chiron et ses disciples et successeurs Machaon et Podalire, qui figurent dans les poèmes homériques. Nous ne parlerons pas ici des personnages réunis sous le nom d'Hippo-

3 Beaumarchais, La Folle Journée, Paris 1785, p. 144. 
CRATE. Mais il nous reste l'étonnant personnage égyptien qu'est Iм'нотеP du règne du roi Zoser, $3^{\mathrm{e}}$ dynastie (2980-2900), médecin, architecte et mathématicien, puis ministre. Plusieurs siècles après sa mort, il obtint la gloire suprême d'être déifié et adoré comme les autres dieux. Nous possédons son portrait ainsi que celui de sa mère KrotioneKH, et sa statuette caractéristique, assise ou debout, se trouve encore à l'heure actuelle en de nombreux exemplaires dans tous les musées égyptologiques - voilà donc une célébrité qui dure depuis 4500 ans. C'est la gloire du premier médecin dont nous connaissions le nom et l'activité; même une œuvre architecturale de sa conception nous a été conservée, la pyramide en degrés du roi Zoser à Saqqâra, une des plus anciennes qui soient, puisqu'elle existait depuis $\mathbf{5 0 0}$ ans, lorsque Abraham naquit à Ur en Chaldée.

Nous pouvons prendre la mesure de ce personnage en lisant (chez Gorce et Mortier), "que chacun a encore ses sentences sur les lèvres», et qu'il aurait rédigé le fameux «chant du harpiste». «Il est le patron des érudits, et aussi des médecins qui le tenaient pour l'inventeur de leur art. Il était encore celui des scribes, qui ne commençaient pas leurs écritures sans lui faire la libation des premières gouttes de leur godet. Les Grecs l'identifiaient à Asklepios. Il entra dans la triade et fut regardé comme le fils de PтAH et de Krotioneкh, une simple mortelle. A la Basse Epoque, il parvint même à supplanter presque entièrement son père dans la faveur populaire.» N'oublions pas pourtant qu'il n'a pas atteint ce but avant que 2500 ans se fussent écoulés depuis son activité dans ce monde.

La Bible ne nous a pas conservé les noms des médecins dont nous apprenons la gloire. Mais un auteur biblique du $\mathrm{II}^{\mathrm{e}}$ siècle avant notre ère, qui ne figure pas dans le «kanon», mais bien dans les livres apocryphes, JEsus, fils de Sirach, dit l'Ecclesiastique, a montré quelles étaient l'estime et la considération dues et accordées au médecin comme tel.

Ce texte pouvant être consulté par chacun, nous ne citerons ici que quelques versets parmi les plus significatifs des chapitres 38 et 39 :

1 «Rends au médecin l'honneur qui lui appartient, pour le besoin que tu en as; car lui aussi a été créé par le Seigneur.

2 Parce que la guérison vient du Très-haut, le médecin sera même honoré des rois.

3 La science du médecin lui fait lever la tête, et le fait admirer parmi les princes.

12 Plusieurs loueront sa prudence, de sorte que sa mémoire ne sera jamais effacée, ni rejetée; mais son renom durera de siècle en siècle.

13 Les peuples parleront de sa sagesse, et toute assemblée célébrera sa louange.

14 S'il demeure, il laisse plus de réputation de sa personne que mille autres; et s'il meurt, il s'acquiert un grand renom.» 


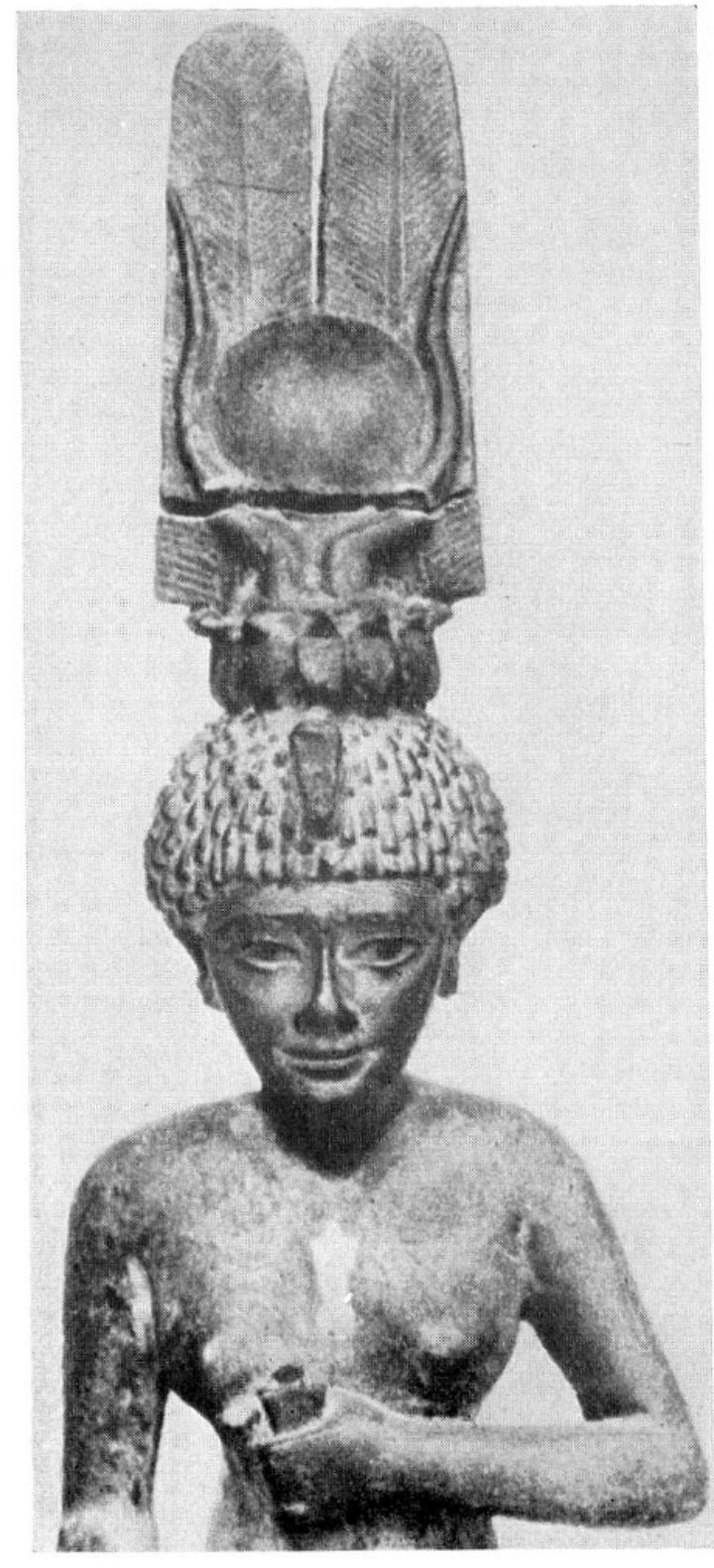

훙

Fig. 1. Krotionekh, mère d'Im'hotep

Gorce et Mortier, Histoire générale des religions, p. 233 


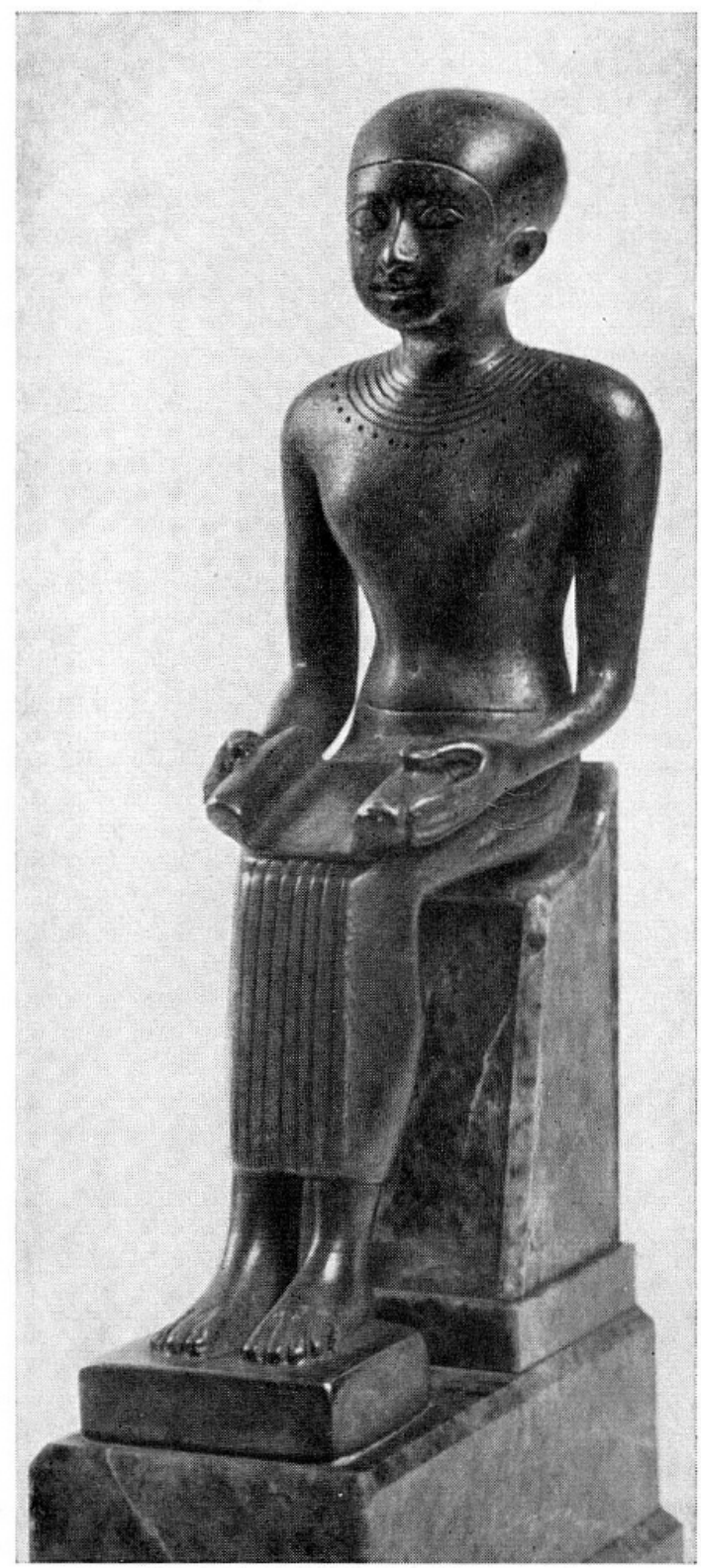

9

Fig. 2. Im'hotep

Statuette d'argent, environ 800 av. J.-C.

«Wellcome» Histor. Med. Museum, No. R 35 / 1936 
Voici terminé le résumé de ce que nous avons trouvé sur la gloire et la célébrité médicales des temps passés. Si nous voulions aussi aborder l'avenir, nous nous en rapporterions à ce que Mr. Chauncey Leake a écrit dans son travail pour le Sigerist-Festschrift, sur le personnage du Gold Rush Doc. «Si l'on est porté à croire que la renommée en médecine ou dans les sciences est méritée par la perfection, on s'apercevra que ce n'est pas le cas. La renommée, tout comme la notoriété, résulte de la publicité, et en bien des cas, l'écart entre elles est fort étroit. Une des joies de la recherche historique consiste précisément à découvrir quelque génie oublié qui mérite d'être rappelé à la mémoire des hommes. Néanmoins, il est difficile de faire place à un nouveau nom dans les rangs serrés de l'histoire qui nous est familière.»

\section{B I B LIO G RAPH I E}

Beld, Sir Charles, Sir Charles Bell's Correspondence with his Brother. London 1870. Bible, Edition David Martin. Bâle 1824.

Brodie, Sir Benjamin Collins, On the Path. \& Surg. of the Diseases of the Joints. London 1819.

- Autobiography in Charles Hawkins, Brodie's Collected Works. 1865.

- Biogr. par Timothy Holmes en Masters of Medicine. 1898.

Beaumarchais, La folle journée ou le mariage de Figaro. Paris 1785, p. 144.

Biographie: August Hirsch, Allgemeines biographisches Lexikon der hervorragenden Arzte aller Zeit und Länder $1884 \mathrm{ss}$.

Bayle et Thillaye, Biographie médicale. Paris 1855.

- Dictionary of National Biography. London 1885-1927.

- Allgemeine deutsche Biographie. München 1875-1900.

Billroth, Theodor, Briefe, hg. von Fischer. Hannover 1896.

Cooper, Bransby Blake, The Life of Sir Astley Cooper. London 1843.

Corvisart, Jean-Nicolas: Hirsch, Allg. biogr. Lexikon ... 1884 ss.

Cruveilhier, J.-B.: Hirsch, Allg. biogr. Lexikon ...

Dieffendach, Joh. Friedrich: Richard Lampe, Joh.-F.Dieffenbach. Leipzig 1934.

Dupuytren, Guillaume: Henri Mondor, Anatomistes et chirurgiens. Paris 1949.

Fothergill, John: R.Hingston Fox, Dr. John Fothergill and his Friends. London 1919.

Frank, Johann Peter: Hirsch, Allg. biogr. Lexikon ...

Frerichs, Theodor von: Naunyn, Erinnerungen, Gedanken und Meinungen. München 1925.

- Hirsch, Allg. biogr. Lexikon ...

Garrison, J.FIELding, An Introduction to the History of Medicin. $4^{\text {th }}$ edition, 1929.

Goldschmid, Edgar, Der Aufschwung in der Medizin der 40er Jahre. Gesnerus, 1949.

Gross, Sam. David, Autobiography. Philadelphia 1887.

Hasse, Karl Ewald, Erinnerungen aus meinem Leben. 2. Auflage, Leipzig 1893.

Hollaender, Eugen, Anekdoten aus der Medizinischen Weltgeschichte. - 3. Auflage, Stuttgart 1950. 
Heim, Ernst Ludwig: Georg Wilhelm Kessler, Der alte Heim. 2. Auflage, Leipzig 1846. Iм'нотеP: GoRCE et MorTier, Histoire des religions. 1948.

Kussmaul, Adolf, Jugenderinnerungen eines alten Arztes. 6. Auflage, Stuttgart 1903.

Lebert, Hermann, Biographische Notizen. Breslau 1869.

Lettsom, John C.: R. Hingston Fox, Dr. Fothergill and his Friends. London 1919.

- James Johnston Abraham, Lettsom, his Life ... London 1933.

Loeffler, Wilhelm, Die Zürcher Medizinische Klinik 1833-1951. Zürich 1951.

Merkel, Friedrich, Jacob Henle. Braunschweig 1891.

Mondor, Henri, Anatomistes et chirurgiens. Paris 1949.

Quincke, Heinrich I.: Edgar Goldschmid, Heinrich Quincke. Schweiz. Medizinische Wochenschrift 44. (1945).

Schönlein, Jo. Lukas: Virchow, Gedächtnisrede. Virchow's Archiv, 1865.

Sigerist, H.E., Große Ärzte. 2. Auflage, München 1933.

Véron, Louis-Désiré: M.-E. BINEt, Un médecin pas ordinaire: Le Dr Véron. Paris 1945.

Virchow, RudolPh: Briefe an seine Eltern. 2. Auflage, Leipzig 1907.

- Hirsch: Allg. Biogr. Lexikon ... Scheuthauer.

Weber, Fred. Parkes, Some Aspects of Death and Correlated Aspects of Life in Art, Epigram and Poetry, $4^{\text {th }}$ edition, London 1922.

\section{Rennward Cysat als Naturforscher, Apotheker und Arzt (1545-1614)}

Von P.Jung, Meggen/Luzern

Der Historiker und Staatsmann Rennward Cysat dürfte allgemein bekannt sein. Hier mögen die wichtigsten Daten dieses selten vielgestaltigen Lebens genügen.

Geboren 1545, als Sproß alten Mailänder Adels, der DE CESATi oder Cisati. Sein Vater war schon in seiner Jugend nach Luzern ausgewandert, heiratete die Tochter des Ritters Rennward GölduIn, dessen Vorfahren aus Zürich kamen, stirbt nach fünf Jahren und hinterläßt eine Witwe mit drei Kindern, darunter RENNWARD. Ausreichende Mittel bestanden keine, die zwei Brüder starben bald, so daß Rennward allein übrig blieb. Anderthalb Jahre nach Vaters Tod geht die Mutter eine zweite Ehe ein mit Conrad von Lauffen. Auch er stirbt bald unbemittelt und hinterläßt seiner Witwe drei Kinder, so daß diese nunmehr die schwere Familienlast mit vier Kindern zu tragen hat.

RenNward entwickelt sich äußerst günstig, fällt durch Begabung und Fleiß auf, lernt als Autodidakt fremde Sprachen, besonders Französisch und Italienisch. Mit vierzehn Jahren, nach sechseinhalb Jahren Schulbesuch, kommt er zu den Apothekern Clauser und Hegner in die Lehre. Er beschäftigt sich viel mit Naturgeschichte, notiert sich bewährte Arzneivorschriften, geht vorhandener ein- 